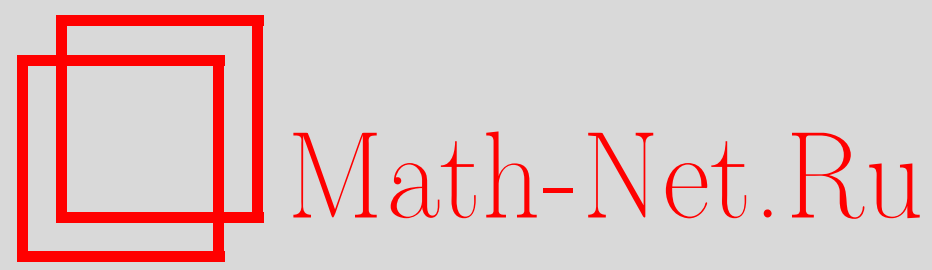

В. И. Рыбаков, Еще один класс пространств Намиоки, Матем. заметки, 2003, том 73, выпуск 2, 263-268

DOI: https://doi.org/10.4213/mzm185

Использование Общероссийского математического портала Math-Net.Ru подразумевает, что вы прочитали и согласны с пользовательским соглашением http://www .mathnet.ru/rus/agreement

Параметры загрузки:

IP: 18.209.158.208

26 апреля 2023 г., 12:43:00

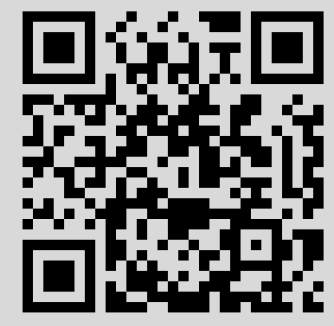




\title{
ЕЩЕ ОДИН КЛАСС ПРОСТРАНСТВ НАМИОКИ
}

\section{В.И. Рыбаков}

\begin{abstract}
Устанавливается принадлежность некоторого класса топологических пространств к классу пространств Намиоки. Результат формулируется в терминах топологических игр.
\end{abstract}

Библиограффия: 7 названий.

Введем некоторые обозначения и напомним необходимые понятия. В заметке линейные пространства рассматриваем над полем $\mathbb{R}$, а топологические пространства считаются отделимыми. Топологическое пространство $T$ назьвают $\mathscr{K}$-счетно-определенным $\mathcal{M}$ если имеется подмножество $S$ в топологическом пространстве $\mathbb{N}^{\mathbb{N}}$ и существует отображение (многозначное) $F: S \rightarrow \mathscr{K}(T)$ ( $\mathscr{K}(T)$ - семейство компактных подмножеств в $T$ ) такое, что для всякого открытого в $T$ множества $U$ множество $\{s \in S$ : $F(s) \subset U\}$ открыто в $S$ и $T=\bigcup\{F(s), s \in S\}$ (см. [1, с. 96]). Если $K$ - компактное множество, то $C(K)$ - пространство вещественных непрерьвных функций на $K$, наделенное sup-нормой; для топологического пространства $T$ обозначим через $C_{p}(T)$ пространство вещественньх непрерьвных на $T$ функций с топологией поточечной сходимости на $T$.

Топологическое пространство $T$ назьвается бәровским, если пересечение всякого счетного семейства всюду плотных открытых в $T$ множеств всюду плотно в $T$.

Будем использовать символ $I$ для обозначения отрезка $[0 ; 1]$. Для элемента $t=$ $\left(t_{\gamma}\right) \in I^{\Gamma}$ (= декартово произведение Г экземпляров отрезка $I$ с тихоновской топологией) обозначим $s(t)=\left\{\gamma \in \Gamma: t_{\gamma}>0\right\}$, а для $Z \subset I^{\Gamma}$ обозначим $s(Z)=\bigcup\{s(t): t \in Z\}$. Положим

$$
\Sigma(\Gamma)=\left\{t \in I^{\Gamma}: s(t) \text { не более чем счетно }\right\} .
$$

Для $\Gamma \subset \Gamma_{1}$ можно вложить $C\left(I^{\Gamma}\right)$ в $C\left(I^{\Gamma_{1}}\right)$ с помошю следующего отображения $p$ : для $u \in C\left(I^{\Gamma}\right)$ положим $(p(u))(t)=u\left(\operatorname{pr}_{\Gamma} t\right), t \in I^{\Gamma_{1}}$ (где $\operatorname{pr}_{\Gamma}-$ каноническая проекция $I^{\Gamma_{1}}$ на $\left.I^{\Gamma}\right)$. В дальнейшем символ $p$ будем опускать и, рассматривая элемент $u \in C\left(I^{\Gamma}\right)$ как функцию из $C\left(I^{\Gamma_{1}}\right)$, будем ее также обозначать через $u$ (из контекста будет ясно, элементом какого пространства рассматривается $u)$.

Введем в этом абзаце обозначения отображений, которыми будем пользоваться на протяжении всего доказательства теоремы. Если $K$ - компактное пространство и $E-$ $\mathscr{K}$-счетно-определенное множество в $C_{p}(K)$, то обозначим через $g_{E}$ непрерывное отображение из $K$ в $C_{p}(E)$ такое, что

$$
\left(g_{E}(y)\right)(h)=h(y), \quad y \in K, \quad h \in E .
$$


Множество $g_{E}(K)$ (рассматриваемое с топологией поточечной сходимости на Е) является компактом Корсона и, следовательно, существует множество $\Gamma_{E}$ и гомеоморфизм $\xi_{E}$ пространства $g_{E}(K)$ на некотороеподпространство в $I^{\Gamma_{E}}$ такие, что $\xi_{E}\left(g_{E}(K)\right)$ $\subset \Sigma\left(\Gamma_{E}\right)$ (см. [2, теорема 3.7] и [3, гл. VI, теорема 7.2, (ii) $\Longrightarrow$ (iii)]). Таким образом, для всякого множества $E$ указанного вида определяются отображения $g_{E}, \xi_{E}$ и непрерьвное отображение $\varphi_{E}=\xi_{E} \circ g_{E}$ пространства $K$ в $\Sigma\left(\Gamma_{E}\right) \subset I^{\Gamma_{E}}$.

Для какого-либо счетного множества $A=\left\{a_{1}, a_{2}, \ldots\right\}$ условимся, что

$$
\sigma_{n}(A)=\left\{a_{1}, \ldots, a_{n}\right\}, \quad n \in \mathbb{N}
$$

Говорят, что топологическое пространство $X$ является пространством Намиоки, если для всяких компактногопространства $K$ и произвольного непрерьвного отображения $f: X \rightarrow C_{p}(K)$ сушествует всюду плотноев $X$ множество $G$ такое, что $f: X \rightarrow C(K)$ непрерьвно в каждой точке из $G$ (см. $[4$, п. 2.2], [5]).

Пусть $\mathscr{P}$ - какое-либо семейство подмножеств топологического пространства $X$. Топологическая игра $G \mathscr{P}$ определяется следуюшим образом (см. [4, п. 2.3]): имеются два игрока $\alpha$ и $\beta$, они поочередно выбирают непустые открытые в $X$ множества. Первым начинает игрок $\beta$, выбирая открытое в $X$ множество $V_{1}$; далее, игрок $\alpha$ выбирает открытое $U_{1} \subset V_{1}$ и множество $A_{1} \in \mathscr{P}$. После того, как каждьй произвел $n$ выборов, $n \geqslant 1$, игрок $\beta$ выбирает открытое $V_{n+1} \subset U_{n}$, а затем игрок $\alpha$ выбирает открытое $U_{n+1} \subset V_{n+1}$ и $A_{n+1} \in \mathscr{P}$ и т.д. Вьиграет $\alpha$, если

$$
\left.\left(\bigcap_{n=1}^{\infty} V_{n}\right) \cap \overline{\left(\bigcup_{n=1}^{\infty} A_{n}\right.}\right) \neq \varnothing
$$

$(\bar{B}$ - замькание в $X$ множества $B)$; в противном случае побеждает $\beta$. Говорят, что пространство $X \beta$-неблагоприятно для игры $G \mathscr{P}$, если игрок $\beta$ не имеет вьшгрьшной стратегии (т.е. какой бы стратегии выбора множеств $V_{n}$ игрок $\beta$ не придерживался, игрок $\alpha$ может так выбирать пары $\left(U_{n}, A_{n}\right)$, что выиграет).

Как установлено в [6, теорема 10], пространство, являющееся $\beta$-неблагоприятньм для игры $G_{\mathrm{a}}$ (где $G_{\mathrm{a}}$ - такая игра $G \mathscr{P}$, когда $\mathscr{P}$ - семейство $\mathscr{K}$-аналитических множеств) будет пространством Намиоки (см. также [4, п. 2.15]).

В нашей заметке устанавливается, что пространством Намиоки является и всякое $X$ из болееширокого класса топологических пространств, именно: когда $X \beta$-неблагоприятно для игры $G_{\mathrm{cd}}$ (где $G_{\mathrm{cd}}$ - топологическая игра $G \mathscr{P}$, когда в качестве семейства $\mathscr{P}$ берется совокупность всех $\mathscr{K}$-счетно-определенных подмножеств в $X$ ).

ТЕоремА. Всякое топологическое пространство, являющееся $\beta$-неблагоприятным для игры $G_{\mathrm{cd}}$, будет пространством Намиоки.

ДоКАЗАТЕЛЬСТво. Пусть $X-\beta$-неблагоприятноепространство для игры $G_{\mathrm{cd}}$. Тогда $X$ является $\beta$-неблагоприятным для игры $G$ (где $G$ - такая игра $G \mathscr{P}$, что семейство $\mathscr{P}$ состоит из одного множества $X$ ); следовательно, $X$ - бэровское пространство [4, п. 2.3]. Возьмем произвольное компактное множество $K$ и какое-либо непрерывное отображение $f: X \rightarrow C_{p}(K)$. Для $\varepsilon>0$ положим

$$
O_{\varepsilon}=\bigcup\left\{U: U \text { открыто в } X \text { и }\left\|f\left(x_{1}\right)-f\left(x_{2}\right)\right\| \leqslant \varepsilon \text { для всех } x_{1}, x_{2} \in U\right\}
$$


(норма в пространстве $C(K)$ ). Если при каждом $\varepsilon>0$ открытое множество $O_{\varepsilon}$ всюду плотно в $X$, то множество $\bigcap_{n=1}^{\infty} O_{1 / n}$, являюшееся всюду плотным (ибо $X$ бэровское), и будет всюду плотным в $X$ множеством точек непрерьвности отображения $f: X \rightarrow C(K)$.

Допустим, что заключение теоремы неверно. Тогда найдутся такое $\varepsilon>0$ и такое непустое открытое в $X$ множество $V$, что $O_{\varepsilon} \cap V=\varnothing$.

Начиная игру $G_{\mathrm{cd}}$, игрок $\beta$ выбирает непустое открытое множество $V_{1} \subset V$ и какую-либо точку $y_{0} \in K$; положим $F_{1}=\left\{y_{0}\right\}$. Пусть в ответ игрок $\alpha$ выбрал открытое множество $U_{1} \subset V_{1}$ и $\mathscr{K}$-счетно-определенное множество $A_{1}$ в $X$. Прежде чем указать следующий выбор открытого множества игроком $\beta$ (которьй следует определенной стратегии игры), проделаем некоторые построения.

Положим $E_{1}=f\left(A_{1}\right)$; множество $E_{1}$ является, очевидно, $\mathscr{K}$-счетно-определенньг в $C_{p}(K)$. Если $g_{1}=g_{E_{1}}$, то функция $g_{1}: K \rightarrow C_{p}\left(E_{1}\right)$ непрерьвна (для всякого $\mathscr{K}$-счетноопределенного в $C_{p}(K)$ множества $E$ еще до формулировки теоремы определены связанные с $E$ отображения $g_{E}, \xi_{E}$ и $\left.\varphi_{E}\right)$ и существуют множество $\Gamma_{1}$ и гомеоморфизм $\xi_{1}=\xi_{E_{1}}$ компакта $g_{1}(K)$ на некоторый компакт в $I^{\Gamma_{1}}$ такие, что $\xi_{1}\left(g_{1}(K)\right) \subset \Sigma\left(\Gamma_{1}\right)$. Пусть $\varphi_{1}=\varphi_{E_{1}}\left(\right.$ напомним: $\left.\varphi_{E_{1}}=\xi_{E_{1}} \circ g_{E_{1}}\right)$ и пусть $J_{1,1}=s\left(\varphi_{1}\left(y_{0}\right)\right)$. Пусть $\Phi_{1}=\left\{\psi_{n}^{(1)}\right.$, $n \in \mathbb{N}\}$ всюду плотно в $C\left(T_{1}\right), T_{1}=I^{J_{1,1}}$. Рассматривая $\psi_{1}^{(1)}$ как элемент из $C\left(I^{\Gamma_{1}}\right)$ (именно: как ранее уже сказано, для $u \in C\left(I^{J_{1,1}}\right)$ и $t \in I^{\Gamma_{1}} u(t)=u\left(\operatorname{pr}_{J_{1,1}} t\right)$, где $\operatorname{pr}_{J_{1,1}}-$ каноническая проекция $I^{\Gamma_{1}}$ на $\left.I^{J_{1,1}}\right)$, определим функцию $\psi^{(1)} \in C(K)$ такую, что

$$
\psi^{(1)}(y)=\psi_{1}^{(1)}\left(\varphi_{1}(y)\right), \quad y \in K
$$

Множество

$$
W=f^{-1}\left(\psi^{(1)}+\left\{h \in C(K):\|h\| \leqslant \frac{\varepsilon}{2}\right\}\right)
$$

замкнуто в $X$. Кроме того, $W$ нигде не плотно в $V$, ибо в противном случае существовало бы такое открытое в $X$ непустое множество $U \subset V$, что $\left\|f\left(x_{1}\right)-f\left(x_{2}\right)\right\| \leqslant \varepsilon$ для любых $x_{1}, x_{2} \in U$, а это противоречит допущению, что $O_{\varepsilon} \cap V=\varnothing$. Вследствие указанных свойств $W$ найдутся $x_{1} \in U_{1} \backslash W, y_{1} \in K$ и $\delta>0$ такие, что $\left|\left(f\left(x_{1}\right)\right)\left(y_{1}\right)-\psi^{(1)}\left(y_{1}\right)\right|=$ $\varepsilon / 2+\delta$.

При этих условиях игрок $\beta$ выбирает теперь непустое открытое в $X$ множество

$$
V_{2}=\left\{x \in U_{1}: \mid(f(x))\left(y_{1}\right)-\left(f\left(x_{1}\right)\left(y_{1}\right) \mid<\frac{\delta}{2}\right\}\right.
$$

в совокупности с конечным подмножеством $F_{2}=\left\{y_{0}, y_{1}\right\}$ в $K$. Очевидно, $\mid(f(x))\left(y_{1}\right)-$ $\psi^{(1)}\left(y_{1}\right) \mid>\varepsilon / 2\left(\right.$ и, следовательно, $\left.\left|(f(x))\left(y_{1}\right)-\psi_{1}^{(1)}\left(\varphi_{1}\left(y_{1}\right)\right)\right|>\varepsilon / 2\right)$ для всякого $x \in V_{2}$.

Пусть далее игрок $\alpha$ выбрал открытое $U_{2} \subset V_{2}$ и $\mathscr{K}$-счетно-определенное $A_{2}$ (можно считать, что $\left.A_{1} \subset A_{2}\right)$. Если $E_{2}=f\left(A_{2}\right)$, то пусть $\varphi_{2}=\varphi_{E_{2}}$ - непрерьвное отображение $K$ в $I^{\Gamma_{2}}$ со значениями в $\Sigma\left(\Gamma_{2}\right)$, заданное подобно тому, как построенное выше отображение $\varphi_{1}$ (которое является композицией двух отображений), при этом множество $\Gamma_{2}$ можно так выбрать, чтобы оно не пересекалось с $\Gamma_{1}$. Положим

$$
J_{1,2}=s\left(\varphi_{1}\left(y_{0}\right)\right) \cup s\left(\varphi_{1}\left(y_{1}\right)\right), \quad J_{2,1}=s\left(\varphi_{2}\left(y_{0}\right)\right) \cup s\left(\varphi_{2}\left(y_{1}\right)\right)
$$

и обозначим через $\Phi_{2}=\left\{\psi_{n}^{(2)}, n \in \mathbb{N}\right\}$ множество, всюду плотное в $C\left(T_{2}\right), T_{2}=I^{J_{1,2}} \times$ $I^{J_{2,1}}$. Пусть $\Phi^{(2)}=\sigma_{2}\left(\Phi_{1}\right) \cup \sigma_{2}\left(\Phi_{2}\right)$. 
Если для $u \in \Phi^{(2)}$ через $u^{(2)}$ обозначить такую функцию из $C(K)$, что

$$
u^{(2)}(y)=u\left(\varphi_{1}(y), \varphi_{2}(y)\right), \quad y \in K,
$$

то, как и вьше, найдем точку

$$
x_{2} \in U_{2} \backslash \bigcup\left[f^{-1}\left(u^{(2)}+\left\{h \in C(K):\|h\| \leqslant \frac{\varepsilon}{2}\right\}\right), \quad u \in \Phi^{(2)}\right] .
$$

В этом случае можно выбрать такое непустое открытое $V_{3} \subset U_{2}$, что для каждого $u \in \Phi^{(2)}$ существует $y_{u} \in K$ такие, что

$$
\left|(f(x))\left(y_{u}\right)-u\left(\varphi_{1}\left(y_{u}\right), \varphi_{2}\left(y_{u}\right)\right)\right|>\frac{\varepsilon}{2}
$$

при любом $x \in V_{3}$.

Игрок $\beta$ выбирает теперь это множество $V_{3}$ вместе с конечньг множеством $F_{3}=\left\{y_{u}\right.$ : $\left.u \in \Phi^{(2)}\right\} \cup F_{2}$ из $K$, а игрок $\alpha$-открытое множество $U_{3} \subset V_{3}$ и $\mathscr{K}$-счетно-определенное $A_{3} \supset A_{2}$.

После выполнения каждым из игроков по $n$ шагов будем иметь: непустые открытые в $X$ множества $V_{1} \supset U_{1} \supset \cdots \supset V_{n} \supset U_{n}$, конечные множества $F_{1} \subset \cdots \subset F_{n}$ в $K$, $\mathscr{K}$-счетно-определенные множества $A_{1} \subset \cdots \subset A_{n}$ в $X$ и $E_{i}=f\left(A_{i}\right), i=1, \ldots, n$, в $C_{p}(K)$.

Тогда, как и вьше, определив множество $\Gamma_{n}$, непересекающееся ни с одним из множеств $\Gamma_{1}, \ldots, \Gamma_{n-1}$, и непрерывное отображение $\varphi_{n}: K \rightarrow \Sigma\left(\Gamma_{n}\right) \subset I^{\Gamma_{n}}$, где $\varphi_{n}=\varphi_{E_{n}}$, положим

$J_{1, n}=s\left(\varphi_{1}\left(F_{n}\right)\right) \subset \Gamma_{1}, \ldots, \quad J_{n-1,2}=s\left(\varphi_{n-1}\left(F_{n}\right) \subset \Gamma_{n-1}, \quad J_{n, 1}=s\left(\varphi_{n}\left(F_{n}\right)\right) \subset \Gamma_{n}\right.$.

Пусть в этом случае $\Phi_{n}=\left\{\psi_{k}^{(n)}, k \in \mathbb{N}\right\}$ всюду плотнов $C\left(T_{n}\right), T_{n}=I^{J_{1, n}} \times \cdots \times I^{J_{n, 1}}$, и $\Phi^{(n)}=\bigcup_{k=1}^{n} \sigma_{n}\left(\Phi_{k}\right)$.

Тогда устанавливается (подобно тому, как и вьше) сушествование такого непустого открытого множества $V_{n+1} \subset U_{n}$, что для каждого $u \in \Phi^{(n)}$ имеется $y_{u} \in K$ такое, что

$$
\left|f(x)\left(y_{u}\right)-u\left(\varphi_{1}\left(y_{u}\right), \ldots, \varphi_{n}\left(y_{u}\right)\right)\right|>\frac{\varepsilon}{2}
$$

при любом $x \in V_{n+1}$. Игрок $\beta$ выбирает теперь это открытое множество $V_{n+1}$ вместе с конечным подмножеством $F_{n+1}=\left\{y_{u}: u \in \Phi^{(n)}\right\} \cup F_{n}$ в $K$. Игрок $\alpha$, в свою очередь, выбирает открытое $U_{n+1} \subset V_{n+1}$ и $\mathscr{K}$-счетно-определенное $A_{n+1} \supset A_{n}$ и т.д.

По условию игрок $\beta$ не имеет вьигрьшной стратегии, поэтому игрок $\alpha$ может так выбирать пары $\left(U_{n}, A_{n}\right)$, что выиграет. В таком случае, существует точка

$$
x_{0} \in\left(\bigcap_{n=1}^{\infty} V_{n}\right) \bigcap\left(\overline{\bigcup_{n=1}^{\infty} A_{n}}\right) .
$$

Положим $E_{n}=f\left(A_{n}\right), J_{n}=\bigcup_{k=1}^{\infty} J_{n, k}, n=1,2, \ldots$, и $E=\bigcup_{n=1}^{\infty} E_{n}$. Множество $E$ является $\mathscr{K}$-счетно-определенным в $C_{p}(K)$, поэтому имеется ранее описанное отображение $g_{E}: K \rightarrow C_{p}(E)$; в качестве соответствующего гомеоморфизма $\xi_{E}$ можно взять следующее отображение:

$$
\xi_{E}(z)=\left(\varphi_{1}\left(y_{z}\right), \varphi_{2}\left(y_{z}\right), \ldots\right) \in \Sigma\left(\Gamma_{1} \cup \Gamma_{2} \cup \cdots\right) \subset I^{\Gamma_{1}} \times I^{\Gamma_{2}} \times \cdots, \quad z \in g_{E}(K),
$$


где $y_{z} \in K$ такова, что $z=g_{E}\left(y_{z}\right)$. Отображение $\varphi_{E}=\xi_{E} \circ g_{E}$ обозначим через $\varphi$.

Семейство функций $\mathscr{F}=\bigcup_{n=1}^{\infty} C\left(T_{n}\right)$ разделяет точки компакта $T=I^{J_{1}} \times I^{J_{2}} \times \cdots$. Действительно, если $t_{1}, t_{2}-$ различные точки из $T$, то существует такой номер $n$, что $\overline{t_{1}} \neq \overline{t_{2}}$ (если $\overline{t_{1}}=\operatorname{pr}_{n} t_{1}, \overline{t_{2}}=\operatorname{pr}_{n} t_{2}$, где $\operatorname{pr}_{n}-$ естественная проекция $T$ на $T_{n}$ ); в таком случае, существует функция $u \in C\left(T_{n}\right)$, которая принимает различные значения в точках $\overline{t_{1}}, \overline{t_{2}}$. Тогда функция $u$ (рассматриваемая теперь как элемент из $C(T)$ ) в точках $t_{1}$ и $t_{2}$ будет принимать различные значения. Так как семейство $\mathscr{F}$ образует подалгебру функций в $C(T)$ и содержит постоянные, то $\mathscr{F}$ всюду плотно в $C(T)$; тогда семейство $\Phi=\bigcup_{n=1}^{\infty} \Phi^{(n)}$ также всюду плотно в $C(T)$.

Если для $y_{1}, y_{2} \in K$ вьполняется равенство $h\left(y_{1}\right)=h\left(y_{2}\right)$ при любом $h \in E$, то, очевидно, вьполняется равенство $\left(f\left(x_{0}\right)\right)\left(y_{1}\right)=\left(f\left(x_{0}\right)\right)\left(y_{2}\right)$; это влечет существование такой функции $u_{0} \in C(\varphi(K))$, что $\left(f\left(x_{0}\right)\right)(y)=u_{0}(\varphi(y)), y \in K$ (естественно, $\varphi(K)$ наделяется топологией, индуцированной из пространства $\left.I^{\Gamma_{1}} \times I^{\Gamma_{2}} \times \ldots\right)$. Пусть

$$
L=\left\{y \in K: s\left(\varphi_{n}(y)\right) \subset J_{n}, n \in \mathbb{N}\right\}
$$

и $M=\varphi(L)$; множество $M$ является компактным подмножеством в $\varphi(K)$. Через $\tilde{u}$ будем обозначать сужение на $M$ функции $u$, определенной на $\varphi(K)$ или на $I^{\Gamma_{1}} \times I^{\Gamma_{2}} \times \cdots$. Семейство $\widetilde{\Phi}=\{\tilde{u}: u \in \Phi\}$, очевидно, всюду плотно в $C(M)$.

Рассмотрим функцию $\tilde{u}_{0}$ ( $u_{0}$ определена выше) и какую-либо функцию $\tilde{u} \in \widetilde{\Phi}$. Пусть $u \in \Phi^{(n)}$. Найдется $y_{u} \in F_{n+1}$ такой, что

$$
\left|\left(f\left(x_{0}\right)\right)\left(y_{u}\right)-u\left(\varphi_{1}\left(y_{u}\right), \ldots, \varphi_{n}\left(y_{u}\right)\right)\right|>\frac{\varepsilon}{2} .
$$

Но $\varphi\left(y_{u}\right) \in M,\left(f\left(x_{0}\right)\right)\left(y_{u}\right)=\tilde{u}_{0}\left(\varphi\left(y_{u}\right)\right)$ и

$$
u\left(\varphi_{1}\left(y_{u}\right), \ldots, \varphi_{n}\left(y_{u}\right)\right)=u\left(\varphi\left(y_{u}\right)\right)=\tilde{u}\left(\varphi\left(y_{u}\right)\right)
$$

следовательно, из (1) заключаем: в нормированном пространстве $C(M)$ расстояние между $\tilde{u}_{0}$ и любым элементом из $\widetilde{\Phi}$ не меньше $\varepsilon / 2$; это противоречит плотности в $C(M)$ семейства $\widetilde{\Phi}$.

Полученное противоречие доказывает, что $O_{\varepsilon}$ всюду плотно в $X$ при любом $\varepsilon>0$ и, тем самым, тогда (как отмечено в начале доказательства теоремы) устанавливается, что отображение $f: X \rightarrow C(K)$ имеет всюду плотное в $X$ множество точек непрерьвности.

Значит действительно, всякое $\beta$-неблагоприятное для игры $G_{\text {cd }}$ пространство является пространством Намиоки.

Следующее утверждение усиливает результат, установленный в [6, следствие 11$]$.

СлЕдСТВИЕ. Всякое бәровское пространство, содерэсащее всюду плотное $\mathscr{K}$ счетно-определенное множество (в частности, всякое сепарабельное бэровское пространство), является пространством Намиоки. 
ДокАЗАТЕЛЬСтво. Пусть $X-$ бэровское пространство и $A$ - всюду плотное в $X$ $\mathscr{K}$-счетно-определенное множество. Рассмотрим игру $G_{\text {cd }}$ на $X$. Пусть $t$ - стратегия игрока $\beta$ в этой игре, и пусть игрок $\alpha$ на $n$ шаге, выбирая пару $\left(U_{n}, A_{n}\right)$, берет $A_{n}=A$, $n=1,2, \ldots$.

Пусть $s$ - такая стратегия игрока $\beta$ в игре $G$ (игра $G$ описана в начале доказательства теоремы), что если в игре $G_{\mathrm{cd}} V_{n+1}=t\left(\left(U_{1}, A\right), \ldots,\left(U_{n}, A\right)\right)$, то $s\left(U_{1}, \ldots, U_{n}\right)=V_{n+1}$. Так как $X$ бэровское, то $X \beta$-неблагоприятно для $G$ (см. $[4$, п. 2.3], либо [7, теорема 2]), поэтому игрок $\alpha$ может так выбирать множества $U_{1}, U_{2}, \ldots$ в игре $G$, что выиграет в этой игре. В таком случае существует $x_{0} \in \bigcap_{n=1}^{\infty} V_{n}$; при этом $x_{0} \in \bar{A}$ (и, значит, $\left.x_{0} \in \overline{\bigcup_{n=1}^{\infty} A_{n}}\right)$. Таким образом, если игрок $\beta$ следует стратегии $t$, то игрок $\alpha$ может выиграть и в $G_{\mathrm{cd}}$; следовательно, $X$ является $\beta$-неблагоприятньг для $G_{\text {сd }}$ и согласно теореме $X$ - пространство Намиоки.

\section{СПИСОК ЦИТИРОВАННОЙ ЛИТЕРАТУРЫ}

[1] Rogers C. A., Jayne J. E. K-analytic sets // Analytic Sets. New York-London: Academic Press, 1980. P. 1-181.

[2] Talagrand M. Espaces de Banach faiblement $\mathscr{K}$-analytiques // Ann. of Math. 1979. V. 110. P. 407-438.

[3] Deville R., Godefroy G., Zizler V. Smoothness and Renormings in Banach Spaces. Pitman Monographs and Surveys. V. 64: Longman Sci. Tech., 1993.

[4] Mercourakis S., Negrepontis S. Banach spaces and topology, II // Recent Progress in General Topology, 1992. P. 493-536.

[5] Debs G. Pointwise and uniform convergence on a Corson compact space // Topology Appl. 1986. V. 23. P. 299-303.

[6] Debs G. Points de continuité d'une fonction séparément continue // Proc. Amer. Math. Soc. 1986. V. 97. № 1. P. 167-176.

[7] Saint-Raymond J. Jeux topologiques et espaces de Namioka // Proc. Amer. Math. Soc. 1983. V. 87. № 3. P. 499-504. 